\title{
Neuro-Muscular Control of Coordinated and Effective Movements: Revisiting Modular Concepts in the CNS
}

\author{
Niladri Kumar Mahato ${ }^{1}$
}

\begin{abstract}
Neurophysiologic analysis of motor behavior has become one of the prime research areas in the domain of Physiology and hence it has seen tremendous development integrated research in this field over the years. This short review discusses the broad approaches which favors to understand effective neural control of motor behavior. The focus of this review is to recognize the gradual evolution of basic ideas regarding execution of coordinated and effective movements. The integrated roles of the spinal cord, the cerebellum and the motor cortex in context of voluntary movements have been delineated with citation of important research observations made in the field of motor control. Internet database related to human motor behavior studies were extensively searched to map the chronological development of important research methods and newer findings in this field. The span of the text ranges from the development of the idea of Motor Primitives to Brain-Machine Interfaces. It is observed that several 'basic' neural modules are preserved through ontogeny and phylogeny. Different combination of hierarchical modular functioning provides a wide range of plasticity required for coordinated and effective skillful movements.
\end{abstract}

Keywords: Brain-Machine-Interfaces; Internal Models; Motor Primitives.

Bangladesh Soc Physiol. 2014, June; 9(1): 42-47

For Authors Affiliation, see end of text.

http://www.banglajol.info/index.php/JBSP

\section{Introduction}

$\neg$ he two classical problems faced by scientists probing motor control and motor behavior in humans included the

Degree of Freedom Problem [DOF] and the problem of Inverse Dynamics. The former issue relates to answering the question that how the motor control system in the body selected the kinematics (relative movements in limb/body segments) of movement to accomplish a given task provided that a task can be completed by adopting a particular strategy that could be selected out of an infinite number of kinematic possibilities to accomplish the same task. The freedom of choice to accomplish a motor task

Received April 2014; $\quad$ Accepted November 2014 was huge and therefore it is perplexing to figure out how the motor system would deal with all the redundancies involved in a motor task. The later problem focused on the issue of selecting segmental muscle forces (kinetics) rather than the movements (kinematics), out of umpteen numbers of possible combinations of timevarying series of muscle forces that can be recruited to accomplish a given motor task. Thus, the aim of the investigators has been fixed to address how the motor system reduces this huge computational load it encounters to decide the course it adopts to construct a particular motor behavior. This review is an effort to overview the ideas that have evolved and have been

J Bangladesh Soc Physiol. 2014, June; 9(1): $42-47$ 
established as fundamental concepts and have helped us define our basic understanding of integrated and coordinated control of motor behavior in humans.

\section{Methods}

Literature related to coordination and effective motor control in humans was obtained from the published material available in the internet. These sources included data and information collected from databases of medical literature like the Medline ${ }^{\circledR}$ and Pubmed ${ }^{\circledR}$ databases. The databases were searched using combinations of several keywords such as kinetics, kinematics, spinal module, motor primitives, internal models, brain-machine-interfaces and some of the other concepts discussed later. Careful efforts were made to choose articles that could help to comprehend the chronology of the evolution of human motor control. Articles consulted for this review were among the pioneering and very frequently cited literature on this particular fields of research.

\section{Discussion}

In the following section, the development of the concepts of the Equilibrium Point hypothesis and Motor Primitives will be discussed. In addition, the development of the concepts of Muscle Synergies and their experimental verification, Internal Models and the role of the cerebellum in refinement of motor control and the evolution of the field of research on the Motor Cortex and Population Coding will be discussed. This section concludes with an overview of the Brain Machine Interfaces and a summarization on the integration and execution of all concepts discussed in the text.

\section{The Physical Plant:}

Researchers, before setting out to define patterns of motor behavior, identified certain properties of the physical plant (the body) that, in a way, restricted or allowed movements of the limbs/ body within limited ranges in the space. These constraints are related to the elastic properties of the tissues, bony configurations at joints and specific arrangement of muscles around the joints that allow defined movements. This partly addressed the redundancy -handling of the central nervous system (CNS) but not the issue of effective motor control that required corrections according to changing physical parameters in the physical environment, for example when faced with the problem of counteracting sudden perturbations of the physical plant. The researchers also visualized that more often than not, movements were executed with segments of limbs as members of a coordinated unit and that these units of behavior were pretty much standardized in terms of fixed action patterns 1 .

\section{Equilibrium Point hypothesis:}

At this juncture of time, the Equilibrium Point (EP) hypothesis was propounded by Feldman et al., in 1976 in the field of kinematic research ${ }^{2}$. This concept seemed to bring motor control understanding into the 'right' perspective. EP suggested that a 'goal' position for a motor task was achieved with limbs assuming an 'EP'. EP emphasized that movements were executed by working through a series of sequentially laid out equilibrium points of the limbs and the joints. It postulated that the elastic properties of the physical plant brought the limb to its EP and the CNS was responsible for setting the lengths and tensions of muscles (physical plant) accordingly to accomplish a motor behavior. Bizzi et al. provided evidence that EP for a task actually worked through a sequence of adjustment of the physical plant, and any sudden imposition and withdrawal of acceleration to the physical plant reinstated the limb segments back to an intermediate EP on its course to accomplishment of the task ${ }^{3}$.

\section{The Motor Primitives:}

Working with spinalised frogs Bizzi and colleagues observed that activation of certain areas on the spinal cord generated specific patterns of motor activation ${ }^{4}$ and stimulation of 
specific grey areas on the cord ventral horns modulated specific movement vectors or movements giving an overall directionality of limbs in space (force fields) ${ }^{5}$. This led to development of the concepts of SPINAL MODULE and MOTOR PREMITIVE [MP] envisaging the existence of functional units in the spinal cord that generated specific patterns of muscle activation ${ }^{6}$. This partly explained the reduction of computational load on the CNS as it was now dealing with modules and not muscles. Experiments also proved that motor commands were not only constituted as linear summation of MPs, but also involved graded/sequential activation and superimposition/overlapping of motor modules in order to generate desired force fields. Any physical obstruction impeding the completion of a motor task was seen to be involved with 'correction' of the force fields with recruitment of required muscle groups to overcome the obstacle ${ }^{7}$.

\section{Concepts of Muscle Synergies:}

Movement scientists at this point speculated and thought that the force fields operated with a group of muscles acting in a fixed balance. This concept defined the new idea of MUSCLE SYNERGY $(\mathrm{MS})^{8}$. Movement was thought to arise from weighted sum of the MS, with the CNS controlling or recruiting a small number of MS coefficients rather than controlling outputs of a large population of individual muscles. MS patterns could be extracted from computational analysis of electromyogram (EMG) clusters from muscles involved in motor behavior. It was very important, also, to notice that activities of MS could be modulated by introducing sensory inputs from the external environment (including the body) into the physical plant ${ }^{6}$. It was observed by Dominici et al. in 2011 that the 'blueprints' of MSs were probably coded in the intermediate horn cells of the spinal cord ${ }^{9}$. They suggested that these basic patterns of motoneuron activities, the motor primitives, were common to and preserved across several species, eventually fine-tuned differently in different species and at different stages of life.

\section{Internal Models and the role of Cerebellum:}

If this were the whole story of motor behavior, how then one accounted for adjustments brought about in motor activity of the physical plant in response to changes in the environment? Could there be certain built-in neural circuitries, the IM that constantly feed the CNS about the changing environment and manipulated the final outcome of motor behavior? Would these sensory inputs act as a basis for a FEEDBACK (delayed) model that is operational just after the appreciation of a change in the environment, or would it be a FEEDFORWARD (fast) model that induces corrections in motor behavior in anticipation (a priori) of any change in the environment, not relying on the information after completion of a motor task. Experiments with transient inertial forces (Corioliis forces) that changed endpoints of movements showed that motor plans could be adjusted moment-to-moment with applications of IMs. IMs received a copy of the motor command, and before the command was physically executed, internal models generated thought that attempted to predict the sensory consequences of carrying out that command in the given state of the physical plant. These predicted sensory consequences were compared to sensory consequences of the desired state, and in case of a mismatch, corrections were introduced to the motor command. Investigators like Sainburg ${ }^{10}$ have indicated the profound role of proprioceptive inputs that went into construction and dynamic restructuring of IMs. Substrates for these models have been shown to predominantly involve the cerebellum that provides an active feed forward estimates required in generation of the predicted sensory consequence, discussed above ${ }^{11-12}$. The cerebellum was active in receiving different modalities of sensation from the external as well as the internal environments, and in coordinating effective motor behavior ${ }^{13}$. There are ample 
evidences indicating that IMs operate in the form of Long-Latency reflexes that assist motor commands in countering unexpected perturbations on the physical plant by calibrating the motor commands as required ${ }^{14}$. Long Latency 4 to be modulated by supra-spinal centers ${ }^{15}$. These long loop reflexes probably induced selective activity in a set of spinal modules to bring about corrections in the motor commands.

\section{Research on the Motor Cortex and Population Coding:}

Fritz and Hitzig reported about the motor cortex in 1873 to be involved with limb movements. Further investigations revealed that areas on the cortex were designated to composite movements involving limb segments rather than controlling individual muscles by Ferrier in 1875. Interestingly, study of evolution of the motor cortex pointed out that some non-mammals actually did not possess definite motor cortex and yet were able to move purposefully in the environment, even in primates, the cortico-spinal tracts (CST) had contributions from the sensory cortex, and CST projected to the dorsal horns in mammals. Frost \& colleagues in 2000 documented graded of separation of the motor cortex from the somatosensory cortex in opossum $^{16}$. The phylogenic development of motor cortex seemed to correlate and coincide with the need for developing better and fine skilled movements, with simultaneous and proportional development of neural substrates required to enhance the quality and a wide array of sensory inputs.

As more of the motor cortex was being explored and deconstructed to understand stimulatory effects of the cortex on the spinal cord, it was getting clear that neurons in the cortex were associated with multi-joint movements in a particular direction (preferred direction). It was proved that the cortical cells (M1 cells) acted in a 'population' activation of clusters of M1 cells and that the final direction of movement in a multijoint system was decided by the weighted vector

J Bangladesh Soc Physiol. 2014, June; 9(1): 42-47 sum of the preferred directions of all the M1 neurons active for that movement ${ }^{17}$. This was called the 'population coding' of movement at the cortex ${ }^{18}$ and demonstrated strong evidence that population coding of M1 neurons mapped specific sets of ethologically relevant postural movements involving sets of muscles, rather than coding for individual muscle activity ${ }^{19}$.

It has been, since then, shown that motor cortical activity (M1 neurons) was involved with reorganization of information related to learning and execution of skilled movements. The motor cortex was the seat for integration of sensory inputs (proprio or exteroception) from other areas of the brain. In this regard it exhibited a lot of 'plasticity' in recruiting additional neurons, devoting them to executing newer learnt tasks (with readjustment of topographic cortical maps), increasing the number of synapses concerned with the newly learnt movement ${ }^{20}$ and in physical addition of the number of dendritic spines in cortical neurons recruited to learn and practice a skilled movement ${ }^{21}$. Not only induction of activity in the cortical M1 cells was involved with integration of information inputs from other areas of the nervous system, but also the activation of specific descending commands via the M1 neurons appeared to extract different muscle synergies at the spinal cord (normal vs. stroke patients) to accomplish a certain task as reported by Cheung et $\mathrm{al}^{22}$. Instrumented activation of M1 neurons have also been seen to activate muscle synergies as a response (in the form of postural adjustment) to sudden perturbation to the physical plant ${ }^{23}$.

\section{Brain Machine Interfaces:}

Latest understanding of functioning of the brain has invested a lot of focus in development of Brain -Machine Interfaces [BMI]. BMIs are systems that recognize the patterns of population coding in the cortex for a required multi-joint movement. The patterns of neuronal firing were recorded from the cortical surface of experimental animals and used to generate real time predictions 
to produce similar movement in a robotic joint. Brain-Machine-Brain Interfaces (BMBIs) are more recent advancements on the BMIs and use artificial sensory inputs into the brain. BMBIs have been successful even in showing corresponding motor cortical activity (in absence of real limb movement) in response to a sensory input. These experimental animals were capable of active exploration of space by moving a virtual $\operatorname{limb}^{24}$. Task accomplishments through these systems have, importantly, demonstrated temporal delay patterns with motor activation only. Simultaneous sensory inputs into the cortex suggest faster and proportionally more precise motor outputs 24 .

\section{Integration and Execution:}

We may thus comprehend that a 'coordinated' and 'effective' movement entails not only static target reaching tasks but also includes momentto-moment dynamic adjustments of the physical plant. Given the existence of anatomical connections between components of the CNS, accurate and skillful voluntary movements were most possibly brought about by (a) sensing environmental cues that acted as inputs to modulate built-in Internal models (orchestrated chiefly in the cerebellum), (b) information fed by the IMs being integrated in the cortex (synaptic modulations) leading to (c) firing from appropriate (preferred directionality) the M1 neurons, that in turn (d) activated pertinent spinal modules and muscle synergies required to accomplish the motor behavior. Different long loop reflexes likely activated specific sets of spinal modules inducing corrections for changing initial conditions in the environment. Temporal EMG latencies associated with such integrated motor behavior point towards simultaneous involvement of multiple areas of the brain towards executing complex motor tasks ${ }^{23-24}$. The MPs, the IMs and cortical M1 activation seem to work simultaneously and mutually to execute effective and meaningful motor behavior with moment-to-moment refining motor output of the physical plant. It can thus be inferred that execution of coordinated dexterous movements integrates the outputs of functional modular behavior from different regions of the CNS. Though lot of the input-output adjustments at the modular levels may seem to be very 'basic' and preserved through ontogeny and phylogeny, combination of hierarchical modular functioning provides a wide range of plasticity for us human beings to train and adapt to requirements of coordinated and effective skillful movements.

To summarize, our understanding of neuromuscular control of coordinated and effective movements has come a long way. The pursuit for better conceptualization of integrated and comprehensive neuro-muscular designs controlling our movements has generated important insight into models of neural control mechanisms of human motion. This knowledge has brought us to the thrones of better analysis of the evolution of movement strategies in man, understanding basic mechanisms underlying adaptations in disease and evolving therapeutic modalities and complex experimental models such as the brain-machine interfaces.

\section{Authors affiliation:}

*1. Niladri Kumar Mahato, Ohio Musculoskeletal and Neurological Institute, Department of Biological Sciences, Ohio University, Athens, Ohio, 45701.812-603-3307; Email: nm620511@ ohio.edu; mahatonk@gmail.com

*Corresponding author

\section{References}

1. Sumbre G, Fiorito G, Flash T, Hochner B. Neurobiology motor control of flexible octopus arms. Nature, 2005; 433:595-596.

2. Feldman AG, Levin MF. The equilibrium-point hypothesis - past, present and future. Adv Exp Med Biol, 2009; 629:699-726.

3. Bizzi, E., Accornero, N., Chapple, W., Hogan, N. Posture control and trajectory formation during arm movement. Behavioral \& Brain Sciences, 1984; 4: $2738-2744$

4. Bizzi E, Mussa-Ivaldi FA, Giszter S. Computations underlying the execution of movement: A biological per- spective, Science,1991; 253: 287-291.

J Bangladesh Soc Physiol. 2014, June; 9(1): 42-47 
5. Bizzi E, Tresch MC, Saltiel P, d'Avella A. New perspectives on spinal motor systems. Nat Rev Neurosci, 2000; 1: 101-108.

6. Bizzi E, Cheung VCK, d'Avella A, Saltiel P, Tresch M. Combining modules for movement. Brain Res Rev, 2008; 57:125-133.

7. Poppele R, Bosco G. Sophisticated spinal contributions to motor control. Trends Neurosci. 2003; 26: 269-276.

8. Tresch MC, Jarc A. The case for and against muscle synergies. Curr Opin Neurobiol, 2009; 19: 601607.

9. Dominici N, Ivanenko YP, Cappellini G, d'Avella A, Mondi V, Cicchese M, et al. Locomotor primitives in newborn babies and their development. Science ,2011; 334:997-999.

10. Sainburg RL, Ghez C, Kalakanis D. Intersegmental dynamics are controlled by sequential anticipatory, error correction, and postural mechanisms. J. Neurophysiol, 1999; 81: 1040-1056.

11. Bastian AJ, Martin TA, Keating JG, Thach WT. Cerebellar ataxia: abnormal control of interaction torques across multiple joints. J Neurophysiol, 1996; 76:492-509.

12. Shadmehr R, Smith MA, Krakauer JW. Error correction, sensory prediction, and adaptation in motor control. Annu. Rev. Neurosci., 2010; 33: 89-108.

13. Gao JH, Parsons LM, Bower JM, Xiong J, Li J, Fox P. Cerebellum implicated in sensory acquisition and discrimination rather than motor control. Science. 1996; 272: 545-546.

14. Kurtzer IL, Pruszynski JA, Scott SH. Long-latency reflexes of the human arm reflect an internal model of limb dynamics. Curr Biol 2008; 18: 449-453.

15. Thilmann A F, Schwarz M, Töpper R, Fellows S J, Noth J. Different mechanisms underlie the longlatency stretch reflex response of active human muscle at different joints. J Physiol. Dec 1991; 444: 631-643.

16. rost SB, Milliken GW, Plautz EJ, Masterton RB, Nudo RJ. Somatosensory and motor representations in cerebral cortex of a primitive mammal (Monodelphis domestica): a window into the early evolution of sensorimotor cortex. J Comp Neurol. 2000 May 22;421(1):29-51.

17. Georgopoulos AP, Schwartz AB, Kettner RE. 1986. Neuronal population coding of movement direction. Science. 233:1416-1419.

18. Schwartz AB, Kettner RE, Georgopoulos AP.. Primate motor cortex and free arm movements to visual targets in three dimensional space. I. Relations between single cell discharge and direction of movement. J Neurosci., 1988; 8:2913-2927.

19. Graziano M. The organization of behavioral repertoire in motor cortex. Annu Rev Neurosci, 2006; 29: 105-134 .

20. Kleim JA, Hogg TM, VandenBerg PM, Cooper NR, Bruneau R, Remple M. Cortical synaptogenesis and motor map reorganization occur during late, but not early, phase of motor skill learning. J Neurosci, 2004; 24: 628-633.

21. Xu T, Yu X, Perlik AJ, Tobin WF, Zweig JA, Tennant $\mathrm{K}$, Jones T, Zuo Y. Rapid formation and selective stabilization of synapses for enduring motor memories. Nature, 2009; 462: 915-919.

22. Cheung VCK, Piron L, Agostini M, Silvoni S, Turolla A, Bizzi E. Stability of muscle synergies for voluntary actions after cortical stroke in humans. Proc Natl Acad Sci, 2009; USA 106: 19563-19568.

23. Pruszynski JA, Kurtzer IL, Nashed JY, Omrani M, Brouwer B, Scott SH. Primary motor cortex underlies multi-joint integration for fast feedback control. Nature, 2011; 478: 387-390.

24. O'Doherty, JE, Lebedev MA, Ifft PJ, Zhuang KZ, Shokur S, Bleuler,H, Nicolelis MA. Active tactile exploration using a brain-machinebrain interface. Nature, 2011; 479: 228 -231. 